http://www.jfas.info

\title{
NATIONAL INSTRUMENTS LABVIEW AND VIDEO IMAGING TECHNIQUE FOR HEALTH STATUS MONITORING
}

\author{
W. L. Khong ${ }^{*}$, N. S .V .K. Rao and M. Mariappan
}

Faculty of Engineering, Universiti Malaysia Sabah, 88400 Kota Kinabalu, Sabah, Malaysia

Published online: 10 September 2017

\begin{abstract}
It is essential to monitor military health status regularly for reducing the risk of cardiovascular diseases (CVD). Physiological parameters such as heart beat rate, blood pressure and electrocardiogram (ECG) features are the most vital indicators for CVD. The standard ECG machines normally used to obtain heart beat rate and ECG features in health facilities are bulky, expensive and the heart's rhythms and activities are recorded on a piece of ECG paper which requires professional cardiologist for interpretation. In this study, National Instruments (NI) LabVIEW Biomedical Workbench with Data Acquisition Device (DAQ) is proposed as an alternative method to receive, save, handle and extract ECG features because of its simplicity, transferability and reliability.
\end{abstract}

Keywords: electrocardiogram (ECG); health monitoring; heart beat rate; NI-LabVIEW; video imaging.

Author Correspondence, e-mail: weileongkhong@gmail.com

doi: http://dx.doi.org/10.4314/jfas.v9i3s.34

\section{INTRODUCTION}

Military which comprises of army, navy and air force is defined as the armed forces authorized by a nation to legally use forces and weapons for defending or attacking perceived 
threats [1]. Military is very important to a nation because they are the protectors to protect citizens from threats and the defense of nation's safety. As a well-trained and professional military, they need to be well prepared in overcoming numerous types of long period strenuous trainings, missions and combats which are highly demands on their physical and mental strength. Hence, health is vital for them to successfully execute the assigned tasks.

According to [12], atherosclerosis is one of the reasons that United States armies died in the Iraq and Afghanistan combats. Atherosclerosis is an early signal of cardiovascular disease (CVD). CVD is referred to a group of disorders related to heart or blood vessels, i.e. arteries, veins and capillaries [3]. In this combat, none of the United States armies had been diagnosed with CVD because all the armies are asymptomatic and feel fine before deployment. This tragedy could happen because high proportions of CVD deaths are occurred without prior signs or symptoms hence CVD also well known as clinically silent disease [4-5]. Atherosclerosis is a condition where plaques, i.e. inflammation, fatty deposits, scar tissues and cells accumulate inside the arteries [6]. As time goes, the plaques will harden and narrow the diameter of the arteries. In the worst situation, when the blood pressure in the blood vessels changed dramatically, the accumulated plaques can suddenly rupture to form the blood clot that able to block the blood flow in the arteries [7]. When the arteries being blocked, the blood will be stopped from flowing to the heart or brain. Once human body organ is not receiving enough amount of blood, the acute events of CVD such as strokes and heart attacks will occur. In 2015, in [8] also claimed that disease and non-combat injury have frequently plagued military campaigns and remain as a major threat to the health of the military. Thus, it is urgently essential to monitor closely on military's health status before, during and after their trainings, missions or combats in order to avoid them from the risk of fatal injuries.

In order to perform a health status diagnosis regarding the CVD, various physiological parameters such as heart beat rate, electrocardiogram (ECG) features, blood pressure and respiratory rate are vital to be measured and analyzed. Among these physiological parameters, ECG features are the principal parameters that commonly used to diagnose CVD because it is the most standard test for cardiac conditions [9-10]. ECG test is a well-established graphical measure technique for recording the bio potential generated by the contraction of heart's 
muscle through the electrodes placing on specific human anatomical i.e. chests, legs, and arms [11]. Usually ECG test services only provided in certain health facilities because ECG machines are extremely bulky and expensive [12-13]. Thus, militaries are hardly carrying along the bulky conventional ECG machine during their deployment or training. Besides, it is also extremely troublesome for the on-duty military to travel a far distance to health facilities for regularly checking their cardiac conditions. Besides, ECG machines only able to record the heart signals on an ECG paper which is lack of self-analyzation and required professional cardiologist to interpret the recorded ECG features [13]. This will lower the chance of the military in regularly checking their health status if they are deployed in combat field, mountain, jungle or ocean for a long period. In addition, ECG signals that recorded on the ECG paper is hard to send for clarification if compared to ECG test method that using computer which can save all the data in soft copy. The ECG signals in soft copy are essential because it can send to the military surgeon when the patient is on the way for treatment or operation. Hence, the first objective of this paper is to demonstrate a computer-based approach which is using National Instruments (NI) LabVIEW Biomedical Workbench with data acquisition (DAQ) device to extract and analyze the ECG signals. NI-LabVIEW Biomedical Workbench had made a great contribution in the area of biomedical engineering by providing users with various types of filtering and signal processing toolkits to extract various physiological parameters sufficiently. Therefore, NI-LabVIEW Biomedical Workbench with the DAQ device is potential as an alternative method for ECG test to overcome the limitations of the conventional ECG machines.

Biomedical engineering is a multidiscipline field that gather the knowledge in engineering, biology and medicine to improve human health care. Over the last few decades, revolutionary technological progress has incited researchers focusing on biomedical engineering to provide significant breakthroughs in diminishing the restrictions on conventional health care monitoring [14]. Hence, computer-based health care monitoring systems have become an inevitable part in biomedical engineering [15]. NI-LabVIEW Biomedical Workbench is a well-known computer-based application for biosignal extraction and analysis. It has a collection of ready-to-run applications, utilities and algorithms to filter biomedical signals 
with advanced signal processing toolkits for simplifying the process of physiological parameters acquiring, storing, processing and extraction. The simplicity, reliability, and transferability of NI-LabVIEW Biomedical Workbench with DAQ device has made it suitable to be frequently used for military's health status monitoring purposes.

NI-LabVIEW Biomedical Workbench with DAQ device is able to extract various physiological parameters accurately, but the wired electrodes that attached to human anatomical are still creating some inconvenient especially when the military is executing task. Besides, only single assessment can be performed per DAQ device at the same time and the reading is better to be taken when the user is in rest position. Hence, the second objective of this paper is to propose a noncontact manner which using video imaging to extract physiological parameter i.e. heart beat rate. Heart beat rate is essential to be measured because it is an indicator for physical conditions and emotional states which are important for the deployment of military [16]. Although video imaging may not be able to provide bioelectric signals that ECG test provides, but the noncontact video imaging technique is able to continuously provide multiple subject's heart beat rate in a comfortable situation and the white coat effects can also be eliminated. Moreover, the captured video can transmit to the military surgeon in advance for investigation and analyzing whether military has irregular or abnormal heart beat rate. If irregular or abnormal heart beat rate is being detected, military surgeon can request the military withdrawn from the deployment or training in order to prevent CVD tragedies happen and further health diagnosis such as ECG test and blood pressure can be carried out. Thus, video imaging with the ability of continuously heart beat rate checking among militaries can be used as an early detection for CVD events.

\section{RESULTS AND DISCUSSION}

Health is indispensable to everyone especially for the militaries who require a lot of strengths and dedication to protect a nation from threats. Thus, it is essential to closely monitor militaries' health status. In this section, various ECG features that extracted from NI-LabVIEW Biomedical Workbench with DAQ device are compared with the ECG test obtained through Philips PageWriter TC30 ECG machine. Fig. 14 indicates the ECG machine 
used in this study which owned by Polyclinic Universiti Malaysia Sabah, and Fig. 15 shows the ECG waveforms and features obtained through ECG machine. Since Lead II ECG waveform is mostly used to continuously monitor cardiovascular status, hence measurements from Lead II are the main focus in this study. Table 1 shows the comparison between the ECG machine and NI-LabVIEW in extracting ECG features. ECG features extracted from NI-LabVIEW can refer to Fig. 1 and Fig. 2.

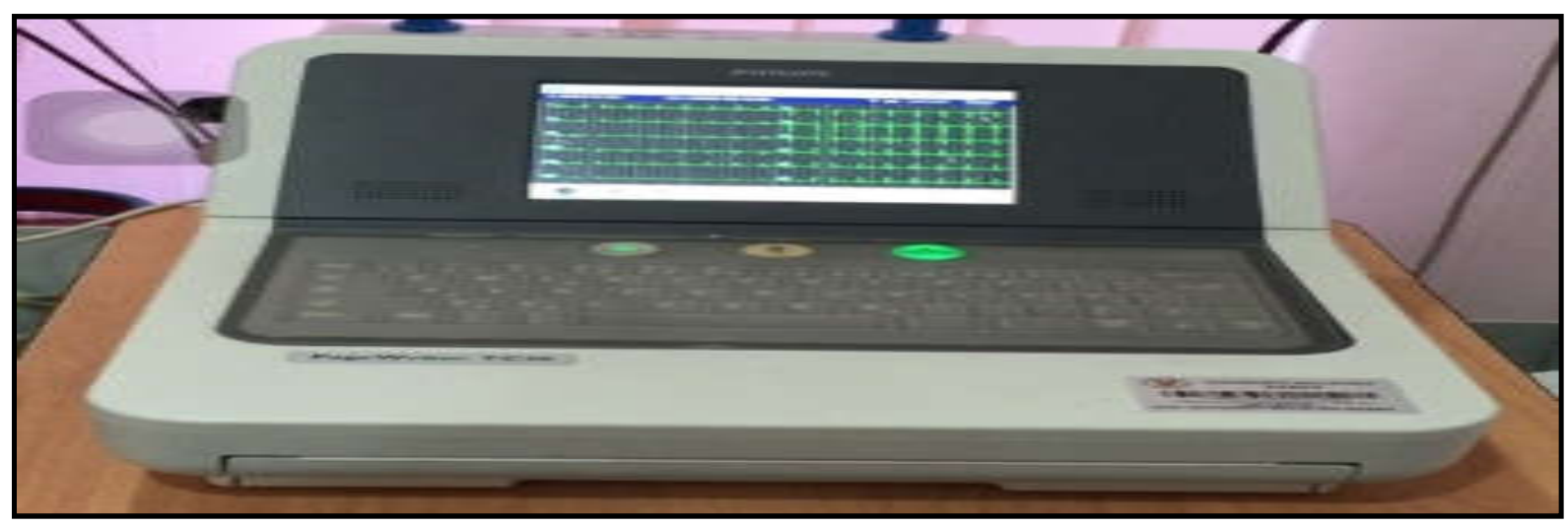

Fig.1. Philips PageWriter TC30 ECG machine 


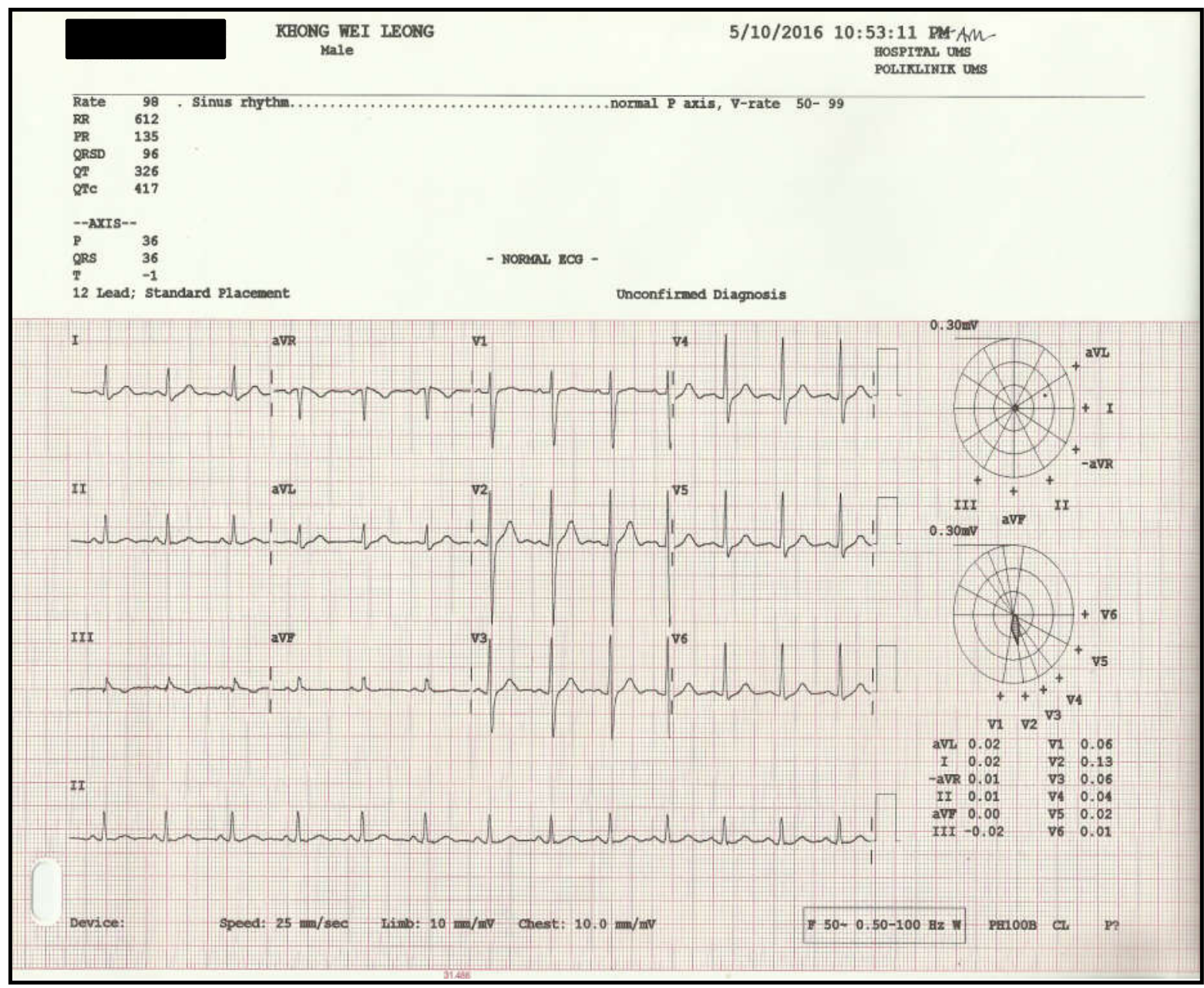

Fig.2. ECG waveforms and features obtained from Philips PageWriter TC30 ECG machine

There are five main ECG features are deliberated in this paper which are the heart beat rate, $R R, P R$ and $Q T$ intervals and $Q R S$ durations. Table 1 shows that all ECG features extracted from NI-LabVIEW Biomedical Workbench are comparable and show a good agreement with the ECG features extracted from ECG machine that used in clinical facilities, which model is Philips PageWriter TC30. Hence, NI-LabVIEW Biomedical Workbench with DAQ device can be one of the alternative methods to acquire ECG features for further analysis if ECG machines are unavailable.

Although ECG machines and NI-LabVIEW Biomedical Workbench can effectively extract ECG features, however, they require wired electrode to obtain those measurements at rest positions. This might become the drawback to the militaries that are on-duty to bring along such machines. Hence, in this study, video imaging technique is proposed to determine human 
heart beat rate in a noncontact manner. Heart beat rate can be used as an initial indicator to monitor the militaries' health status. If military's being detected has irregular, unusual or reached maximum heart beat rate, they can withdraw from tasks for immediately conducting ECG test to diagnose their cardiovascular conditions. Since ECG features extraction using NI-LabVIEW Biomedical Workbench with DAQ device show a good agreement with the clinical used ECG machine (Philips PageWriter TC30) which only $1 \mathrm{bpm}$ difference for the heart beat rate detection, hence NI-LABVIEW is used to verify the heart beat rate extracted through video imaging technique. Table 2 shows the subjects' heart beat rate extracted through video imaging technique and compared with the heart beat rate extracted from NI-LabVIEW Biomedical Workbench.

Table 1. Comparison Between Philips PageWriter TC30 ECG machine and NI-LabVIEW Biomedical Workbench with DAQ device in extracting ECG features

\begin{tabular}{ccccc}
\hline ECG Features & Philips PageWriter TC30 & NI-LabVIEW & Difference & \% Difference \\
\hline Heart Beat Rate & $98 \mathrm{bpm}$ & $97 \mathrm{bpm}$ & $1 \mathrm{bpm}$ & $1.0 \%$ \\
RR interval & $612 \mathrm{~ms}$ & $631 \mathrm{~ms}$ & $19 \mathrm{~ms}$ & $3.1 \%$ \\
PR interval & $135 \mathrm{~ms}$ & $121 \mathrm{~ms}$ & $14 \mathrm{~ms}$ & $10.4 \%$ \\
QT interval & $326 \mathrm{~ms}$ & $325 \mathrm{~ms}$ & $1 \mathrm{~ms}$ & $0.3 \%$ \\
QRS duration & $96 \mathrm{~ms}$ & $110 \mathrm{~ms}$ & $14 \mathrm{~ms}$ & $14.6 \%$ \\
\hline
\end{tabular}

*Note: bpm-beat per minute; ms-miliseconds. 
Table 2. Subjects' heart beat rate extraction through video imaging technique and compared with the results obtained from NI-LabVIEW Biomedical Workbench

\begin{tabular}{ccccccc}
\hline Age & Gender & $\begin{array}{c}\text { NI-LabVIEW } \\
\text { (ref. BPM) }\end{array}$ & $\begin{array}{c}\text { Video Imaging } \\
\text { Technique }\end{array}$ & $\begin{array}{c}\text { Diff. } \\
(\mathbf{B P M})\end{array}$ & $\begin{array}{c}\text { Error } \\
(\mathbf{\%})\end{array}$ & $\begin{array}{c}\text { Accuracy } \\
(\%)\end{array}$ \\
\hline 13 & Female & 86 & 86 & 0 & 0 & 100 \\
31 & Male & 77 & 75 & 2 & 2.6 & 97.4 \\
31 & Male & 74 & 73 & 1 & 1.4 & 98.6 \\
31 & Male & 74 & 77 & 3 & 4.1 & 95.9 \\
31 & Male & 97 & 96 & 1 & 1.1 & 98.9 \\
31 & Female & 80 & 80 & 0 & 0 & 100 \\
52 & Female & 68 & 68 & 0 & 0 & 100 \\
57 & Male & 86 & 83 & 3 & 3.5 & 96.5 \\
81 & Female & 64 & 70 & 6 & 9.4 & 90.6 \\
\hline
\end{tabular}

Volunteers that participate in this study are from 13 years old to 81 years old. The participants with a wide range of age are chosen in this study is to investigate the robustness of video imaging technique for extracting subject's heart beat rate under different skin roughness which caused by aging factor. According to American National Standard ANSI/AAMI EC13:2002 developed by the (Association for the Advancement of Medical Instrumentation, 2002), the minimum required range and maximum allowable error for heart beat rate measurements are $\pm 5 \mathrm{bpm}$ or $\pm 10 \%$, whichever is greater. In addition, in [40] stated that heart beat rate measurement with error within $\pm 5 \mathrm{bpm}$ is probably to be acceptable especially for the measurements can be performed remotely. Hence, Table 2 shows that the heart beat rate extracted through video imaging technique are comparable and reached a good agreement with NI-LabVIEW Biomedical Workbench by achieving more than $90 \%$ of the accuracy which is less than $10 \%$ in the readout error for all the participants. The results also show that the 81 years old subject's heart beat rate extraction through video imaging technique has 6 bpm differences with the results of NI-LabVIEW Biomedical Workbench, this might due to the wrinkles on the skin are influenced the results. However, the readout error is still able to keep below the margin set by American National Standard which is $\pm 10 \%$. From the results, 
it indicates that heart beat rate is possible being extracted from human skin through video imaging technique. Thus, it can be applied to determine military's heart beat rate remotely for determining any irregular, abnormal or maximum heart beat rate during and/or before their deployment, training or at rest. Militaries can proceed for ECG test with NI-LabVIEW Biomedical Workbench in a timely manner if ECG machines are unavailable when irregular or abnormal heart beat rate situations are occurred. So, the military's health status can be monitored closely to avoid the sudden cardiac deaths and to make sure the militaries that assigned for deployment are in a healthy condition.

\section{EXPERIMENTAL}

ECG is a measurement test for heart's bio electrical activities by plotting the results on ECG graph paper. These electrical activities are controlled by myocardial cells which responsible to regulate the physical contraction of the heart muscle. These myocardial cells exhibit four unique properties, i.e. automation, excitation, conduction and contraction [17]. Automation means the myocardial cells generate electrical impulses spontaneously to control the heart contraction rhythmic automatically. Excitation is defined as the state of myocardial cells in responding to an electrical impulse. Conduction is referred as myocardial cells receive an electrical impulse and subsequently transfer it to adjacent cells. Meanwhile, contraction is the myocardial cells are being shortened to cause the cardiac muscle contract. The rhythmic contractions of cardiac muscle will produce the electrical impulses that can be measured through ECG test. Based on these four properties, myocardial cells can be further categorized into two types: (i) myocardial cells that capable of contraction and (ii) myocardial cells that capable of conduction [18]. These myocardial cells contain contractile protein which has the ability to contract and subsequently go back to their original length to produce the mechanical pumping action of the heart. The repeated pumping actions of the heart make myocardial cells polarize, depolarize and repolarize when an impulse develops and spread throughout the myocardium. Waves of repolarization and depolarization represent the bio electrical activities of the heart that is capable to be detected by placing electrodes on the human body and the recorded ECG waveform is printed on a piece of ECG graph paper by the ECG machine. The 
normal ECG waveform is comprised of a sequence of positive and negative $\mathrm{P}$ wave, QRS complex and $\mathrm{T}$ wave as shown in Fig. 3. The $\mathrm{P}$ wave shown in Fig. 1 denotes the first atrial depolarization; QRS complex denotes the ventricular depolarization and T wave denotes the repolarization of the ventricles so the myocardial cells regain the negative charges and the atrial can depolarize again [19].

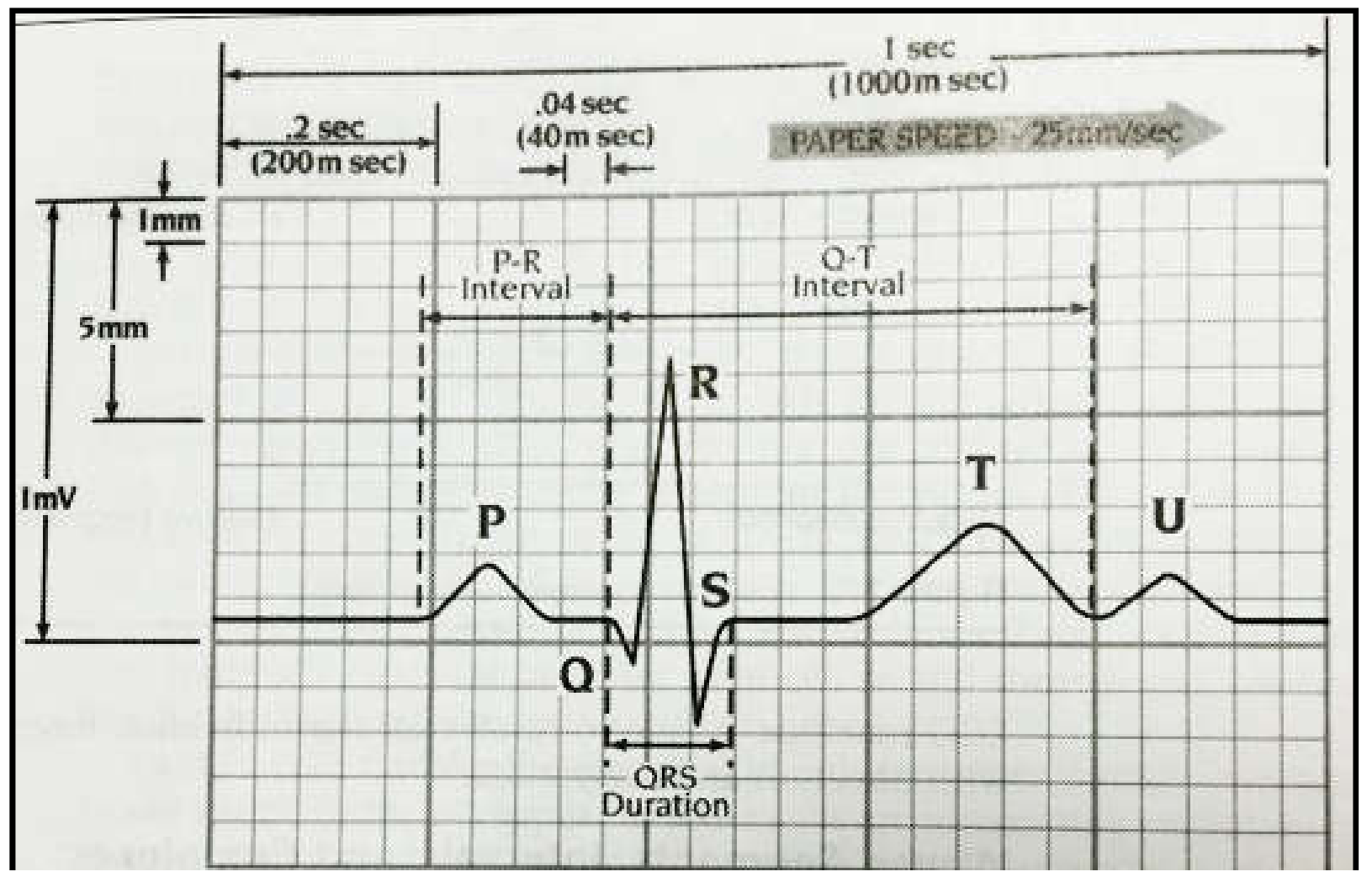

Fig.3. The normal orientation of ECG waveform recorded and ECG paper with grids [18]

The electrode used to receive electrical impulses generated by heart is an adhesive pad that contains conductive gel that designed can be attached to human skin. Conductive gel is used to enhance the trace quality of the bio potential for producing a better ECG signals. The electrodes are then connected to the ECG machine by wires are called leads wire. Fig. 4 shows the electrodes and leads wires that usually used in health facilities. According to [20], the placement of electrodes on the human body can be categorized to limb leads and precordial (chest) leads (V1-V6). However, limb leads can be further divided into bipolar limb leads (Lead I, II and III) and unipolar limb leads (aVR, aVL, aVF). In these 12-leads ECG placements, Lead II is the most preferred monitoring lead of choice and most commonly used for continuous ECG monitoring because it can give a positive deflection [17, 21-22]. 
Therefore, Lead II is used in this study due to its benefits.

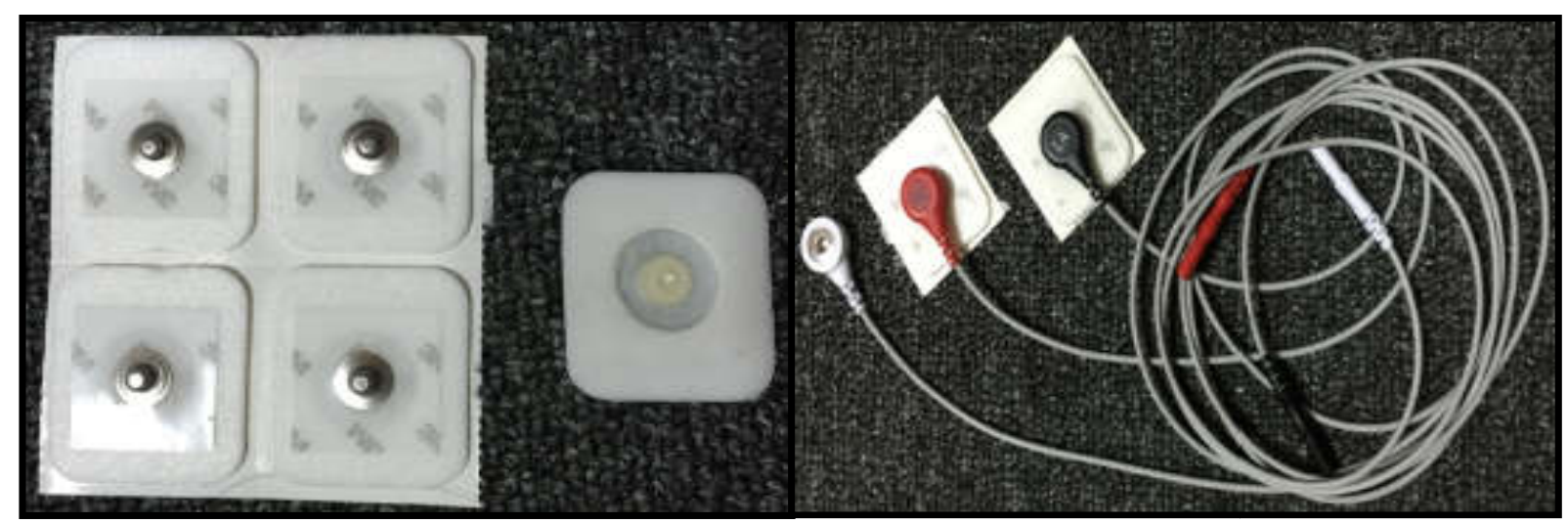

(a)

(b)

Fig.4. (a) Electrodes used in clinical. (b) Lead wires used for bipolar limb leads

Lead II is one of the standard bipolar limb leads. The arrangement of electrodes on human body is based on Einthoven's triangle, it is a triangle arrangement with the heart at its center which surrounded by right arm (RA), left arm (LA) and left leg (LL) as shown in Fig. 3 [23]. Bipolar is referred as the bio potential at one electrode is compared with another electrode, hence it is only involved two leads when gather the information of the electrical activities [24]. As a precaution, it must beware to the polarity when connect the electrodes to an ECG machine for measuring the bio potential difference between the two electrodes. Lead II used in this study is to measure the electrical potential difference between RA and LL where RA is the electrode with the negative terminal and LL is the electrode with the positive terminal as shown in Fig 5.

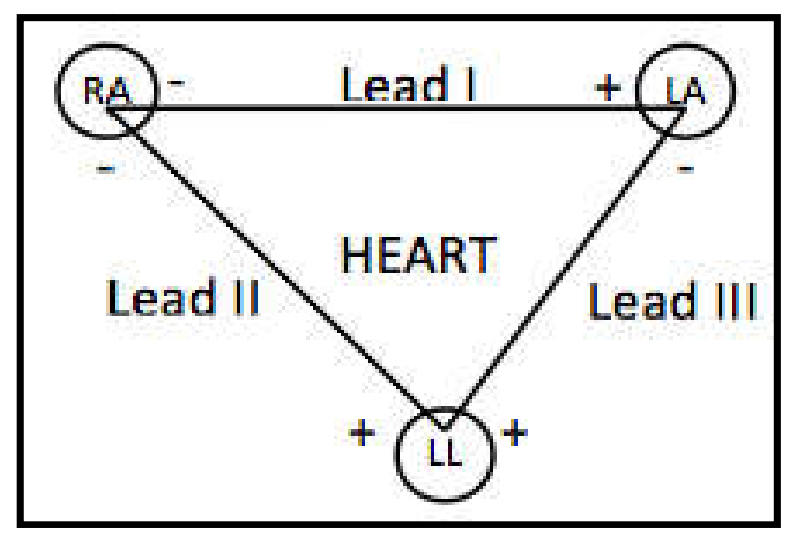

Fig. 5. Bipolar limb leads placement based on Einthoven's Triangle

\subsection{National Instruments (NI) LabVIEW}

LabVIEW is a graphical coding design platform developed by National Instruments (NI) [25]. 
The advantage of LabVIEW over other platform is its ability in supporting the assessment of instrumentation hardware i.e. DAQ device. The main function of the DAQ device is acts as the interface to connect the computer with the outside environment through digitizing the received analog signals for computer to further analyze the signals. A DAQ device normally consists of counter, analog and digital I/O. There are a wide range of different model DAQ devices are available from NI. Hence, the user can choose the appropriate DAQ device to match with their research purposes.

In this study, NI USB-6281 DAQ device is used to acquire the electrical potential from the electrodes placed on the human body. The placements of the electrodes are based on Lead II connection. NI USB-6281 is a NI M-series with high accuracy multifunctional USB DAQ device that is optimized for high accuracy measurement at high sampling rates. It is a DAQ device with 16 analog inputs (18 bits) associated with maximum rate of $625 \mathrm{kS} / \mathrm{s}$ per single channel, 32 bits counters and 2 analog outputs (16 bits) associated with maximum rate of 2.8 MS/s, 24 digital I/O (8 clocked). This high accuracy provided by NI USB-6281 DAQ device make it able to acquire data from analytical instrumentation and/or medical devices [26]. The 18 bits accuracy of DAQ device also benefits the portable data logging function because it helps logs voltage data rapidly and conveniently, which is very vital in acquiring ECG signals. Fig. 6 shows the NI USB-6281 DAQ device with all its labelled screw terminal pinout. 


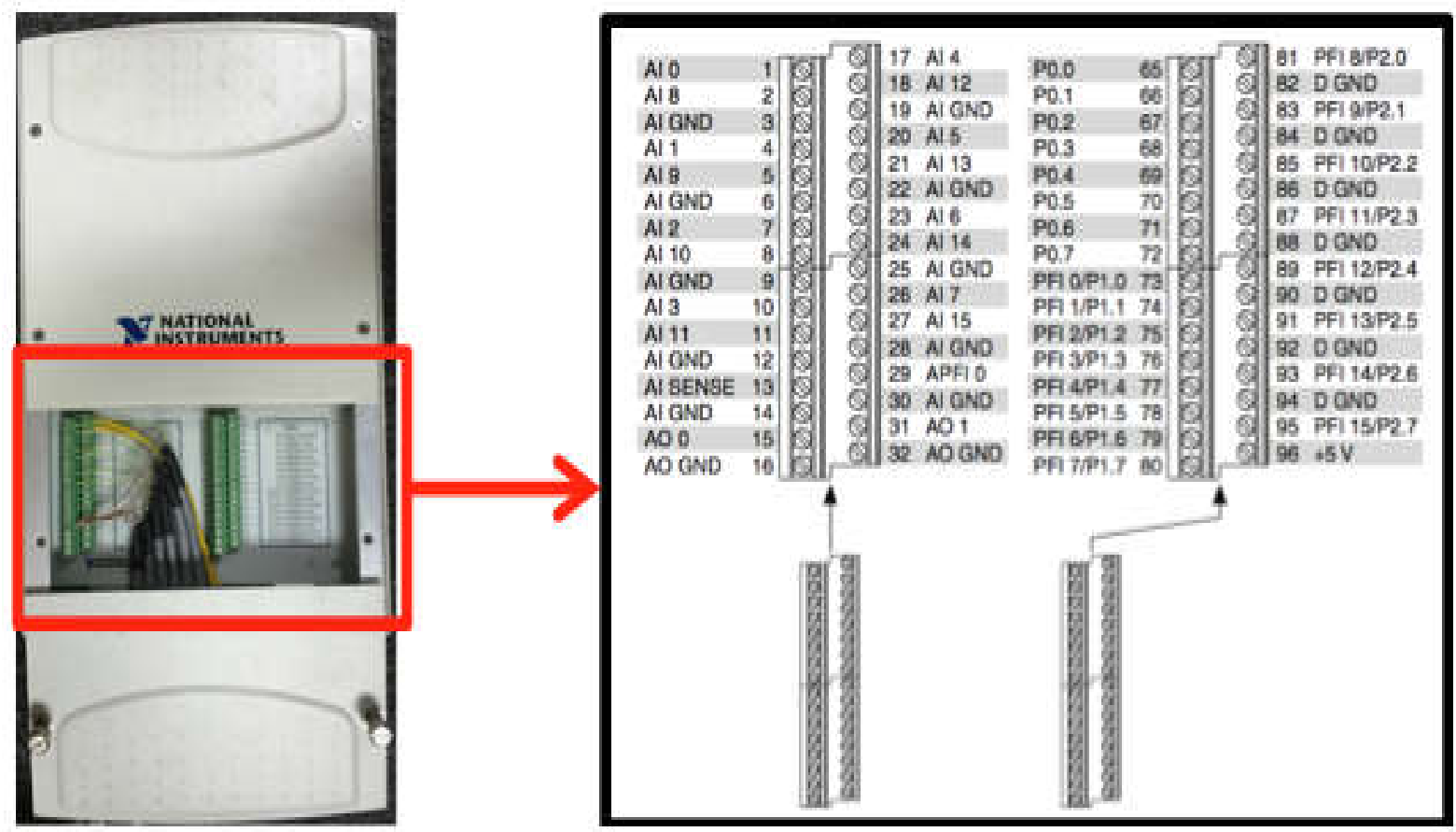

(a)

(b)

Fig.6. (a) NI USB-6281 DAQ device. (b) NI USB-6281 labelled screw terminal pinout[27]

NI USB-6281 DAQ device contains three types of analog input (AI) ground reference settings which are differential mode (DIFF), referenced single-ended mode (RSE) and non-referenced single-ended mode (NRSE) [27]. In this study, Lead II of ECG measurements is proposed to continuously monitor the health status which is a bipolar limb lead. Hence, DIFF mode is used to measure the difference in voltage between two AI signals. Moreover, the bio potential generated by heart is very low also fulfill the condition of DIFF mode that is the input signal is low level with less than $1 \mathrm{~V}$. On the other hand, the input signal for RSE mode and NRSE mode is greater than $1 \mathrm{~V}$. Thus, RSE and NRSE mode are not suitable for measuring heart activities in this study because the measurements involve only in millivolts. For differential measurements, AI 0-AI 7 and AI 8-AI 15 are the groups of positive and negative analog inputs respectively, thus it must pair the analog input accordingly in order to measure the voltage difference. In this study, a pair of analog input (AI 4 and AI 12) is used to acquire Lead II ECG signals. AI 4 (positive terminal) is connected to electrode at LL. Meanwhile, AI 12 (negative terminal) is connected to electrode at RA.

After the connections of Lead II had been set up in DAQ device, NI-LabVIEW biomedical 
Workbench with various applications is used to receive, save, handle and extract the ECG signals measured through DAQ device. Fig. 7 shows the ready-to-run applications in NI-LabVIEW biomedical Workbench which consists of biosignal logger, viewer and generator, ECG feature extractor, heart rate variability analyzer, non-invasive blood pressure analyzer, file format converter, 3D reconstructor, biosignal file manager, biosignal calibrator and biomedical utility tool. Since ECG signals are the main focus in this study to monitor health status, and hence biosignal logger and viewer and ECG feature extractor are presented in this paper.

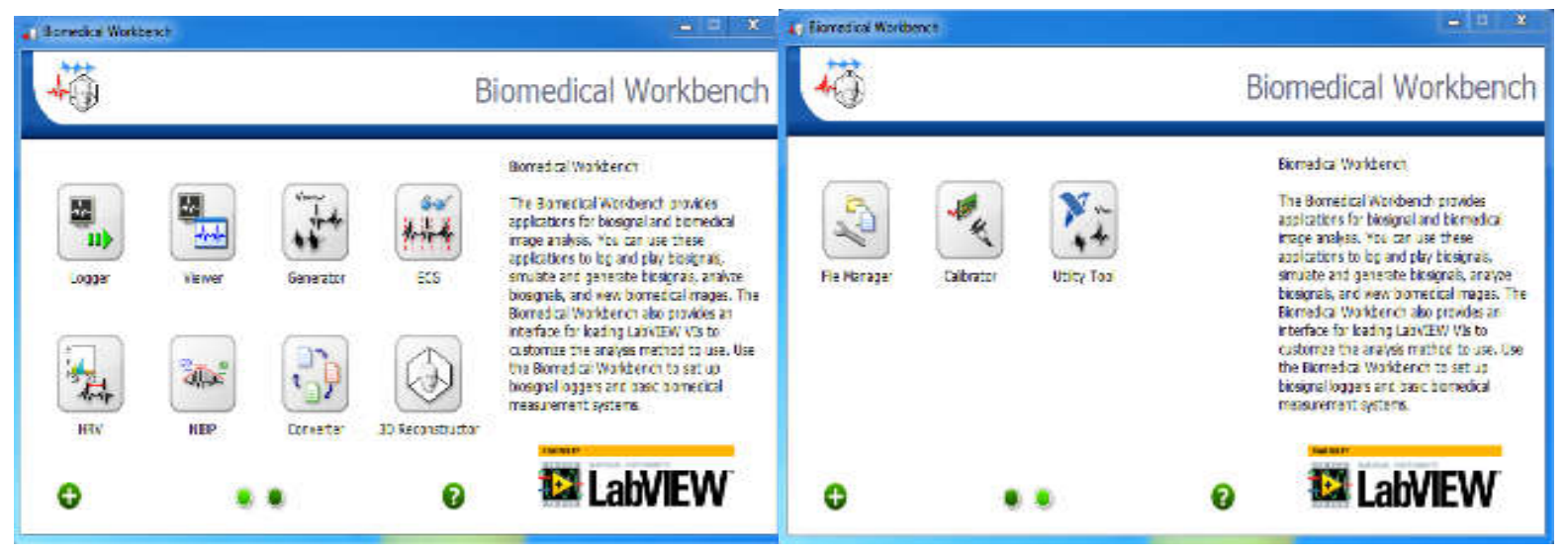

Fig.7. Ready-to-run application in NI-LabVIEW Biomedical Workbench

Biosignal logger and viewer application in NI-LabVIEW Biomedical Workbench is consists of two parts which are biosignal viewer and biosignal logger. The function of biosignal viewer is to replay the biosignal files being saved previously. Meanwhile, biosignal logger is used to connect NI-LabVIEW DAQ device with sensors or electrodes for logging biosignals. It uses analog input channels of DAQ device to obtain the biosignals. In this study, ECG signals are being logged for saving, processing and extraction purposes because ECG waveform is the principal parameters that commonly used to diagnose CVD and to monitor health status.

After biosignal logger and viewer application is launched, the application will request the user to choose the corresponding DAQ device. Subsequently, continuous samples option is selected for the acquisition mode to acquire the ECG signals. According to [28], the typical sampling rates for acquiring ECG signals from the NI DAQ devices are $125 \mathrm{~Hz}$ and $250 \mathrm{~Hz}$. $250 \mathrm{~Hz}$ is selected in this study because modern ECG devices are operate at this frequency in recording heart rate variability [29]. Next, differential option is chosen for the terminal configuration due to the Lead II connection. 
NI LabVIEWbiosignal logger also allows the user to create virtual channels to filter and customize the biosignals that user wants to log by providing various types of digital filter operation such as notch, math, adaptive and derivative/integral/moving digital filter. There are various types of noises could contaminate the logged ECG signals such as power line interference, electrode and electromyographic (EMG) [41] noise, motion artefacts and baseline noise wandering. Among these noises, power line interference is the most significantly noise that affects the ECG signals [31-31]. Power line interference is a type of noise with high frequency that usually centered at $50 \mathrm{~Hz}$ or $60 \mathrm{~Hz}$ with a bandwidth of less than $1 \mathrm{~Hz}$. The power line interference noise is created because the nearby devices could interfere the measurement when the ECG device is inappropriate grounded [32]. To overcome the power line interference, notch filter with centered frequency at $60 \mathrm{~Hz}$ and bandwidth of 1 $\mathrm{Hz}$ are used in this study to pre-process the ECG signals obtained from the NI-LabVIEW DAQ device before sending to ECG Feature Extractor to extract the ECG features from the signals. Fig. 6 indicates the configuration settings for virtual channel to filter the power line interference. The ECG signals obtained from biosignal logger are saved in a binary measurement file, which format is .tdms. Due to the advancement of telemedicine technology, the data saved in soft copy can easily transmit to cardiologist for interpreting and investigating purposes. The ECG signals in soft copy are vital especially for the militaries that are deployed far away from the health facilities because the medical information can exchange from one site to another easily, which is essential in enhancing the military's health status. 


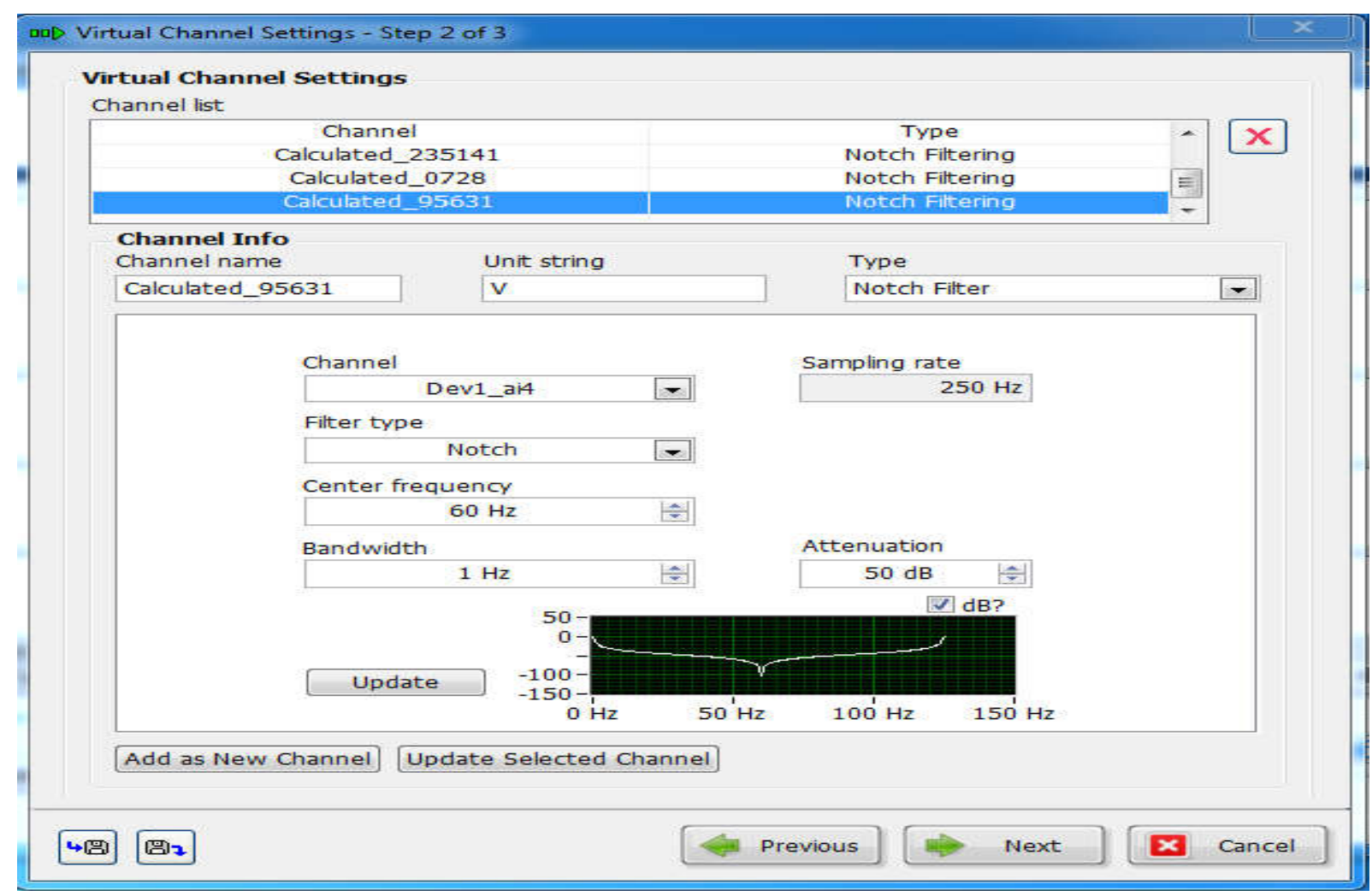

Fig.8. Virtual channel setting in NI-LabVIEW ECG feature extractor to filter power line interference

ECG feature extractor in NI-LabVIEW Biomedical Workbench is an application for extracting ECG features. ECG Feature Extractor acquires ECG signals through two ways: (1) acquiring real time ECG signals from the DAQ device and (2) importing ECG signals from various data sources. File formats that are supported by ECG feature extractor are TDM (.tdm), TDMS (.tdms), MATLAB (.mat), PhysioBank Database (.hea), LVM (.lvm), HL7_aECG (.h17), Axon Instruments data file formats (.abf), European Data Format (.edf\& .rec), iWorkx (.iwxdata), CSV (.csv), EMGWorks (.emg), AstroMed (.dcr), SCP (.scp), C3D (.c3d), ACQ (.acq), DDF (.ddf) and Audio files format (.mp3, .wma \& .wav) [33].

In order to diagnose health status, ECG features such as QRS intervals, QRS amplitudes, PR intervals, QT intervals and etc. need to be extracted from the preprocessed ECG signals. The mentioned features provide information about heart beat rate, heart conduction and contraction velocity and heart abnormalities which can be used for the diagnosis of CVD. Due to this fact, it has drawn attention in the ECG signal processing field especially for the militaries who require a very healthy condition to protect the nation from perceived threats. In ECG Feature Extractor, user can choose whether want to detect QRS wave only or to detect 
all ECG features. The ECG feature extractor is starts by detecting the entire $\mathrm{R}$ wave in the ECG signals; next other ECG features for every beat only will be extracted. Hence the ability in detecting $\mathrm{R}$ waves is very vital for ECG Feature Extractor. The R waves are easily being detected if the user's ECG waveform is in normal condition. However, if the user with some specific heart disease, the extraction of abnormal ECG waveform will be difficult. Hence, the ECG waveform is better undergoing preprocessing process for enhancement before the features being extracted. After preprocessed the entire ECG signals, the exact position of the $\mathrm{R}$ waves will be more clearly and easily being identified.

$\mathrm{R}$ waves in human ECG signals usually have a frequency between $10 \mathrm{~Hz}-25 \mathrm{~Hz}$ [34-35]. Therefore, ECG Feature Extractor provides a bandpass filter for the user to filter ECG signals so that R waves in the entire ECG signals are more obvious and clearly being identified. Fig. 7 shows feature settings to filter ECG signals. Besides, ECG feature extractor also provides rectifier for the users to further enhance the ECG signals in detecting the $\mathrm{R}$ waves by using absolute or square method. Fig. 8 indicates filtered ECG signals with bandpass frequency 10 Hz-25 Hz. After the R waves being detected, other features will be extracted accordingly by using $\mathrm{R}$ waves as the references. Heart beat rate (HBR) can be determined through the RR interval (RR) by using Equation (1). Besides, PR interval extracted from ECG feature extractor can also be used to calculate the duration for the atrial starts to depolarize until ventricular starts to depolarize. Moreover, QRS complex is used to represent the duration for the ventricular depolarize. QT interval is used to calculate the time required for ventricular to depolarize and repolarize again. All extracted ECG features are displayed in statistics as shown in Fig. 9. After the features being extracted, users can further analyze the RR intervals through heart rate variability analyzer application provided in NI-LabVIEW Biomedical Workbench to demonstrate the state of the heart and nerve system as indicated in Fig. 10. Thus, militaries able to evaluate their general status of heart and nerve system based on these measurements obtained through NI-LabVIEW Biomedical Workbench before consult the military surgeon.

$H B R=\frac{60}{R R}(1)$ 


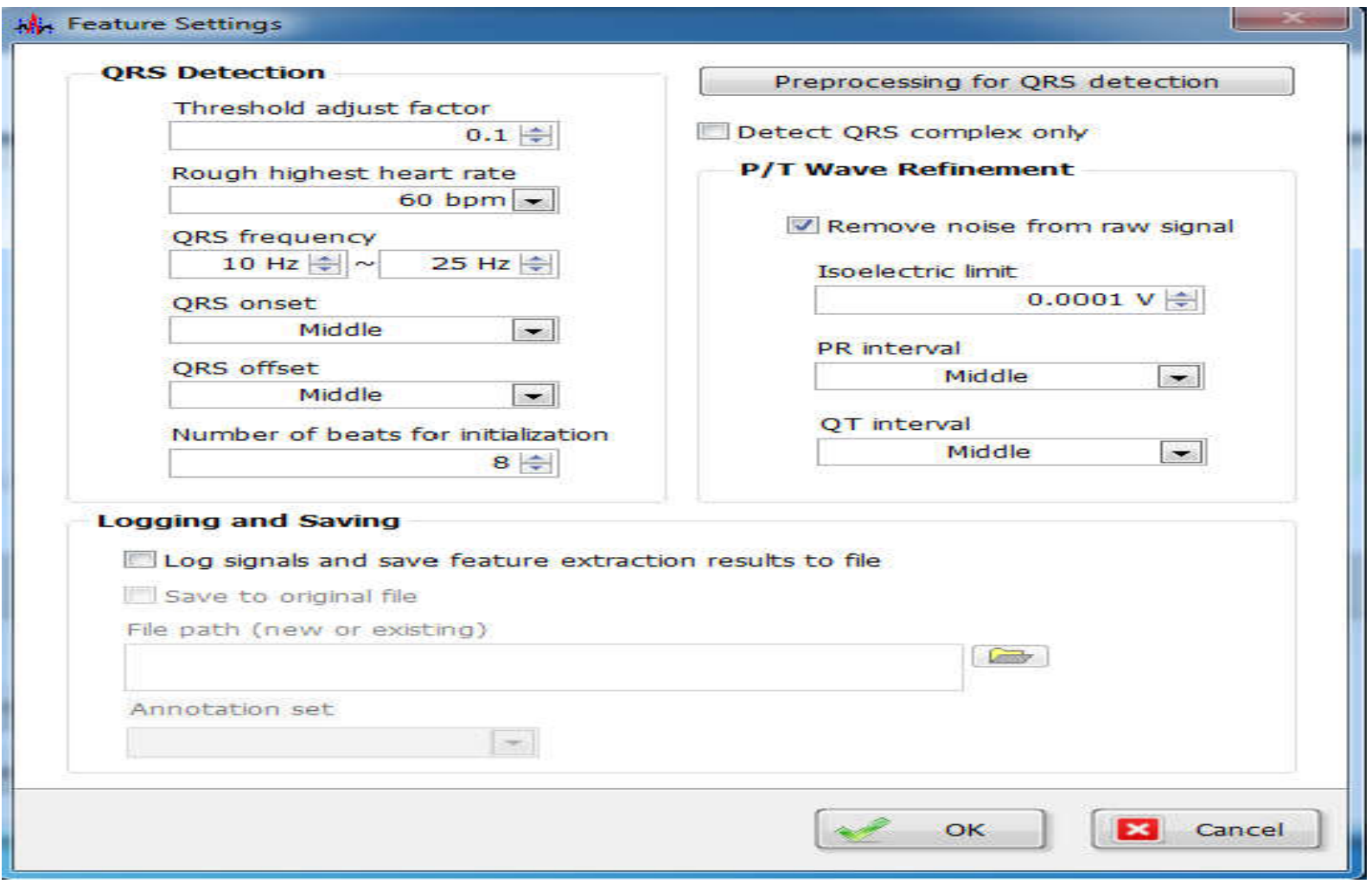

Fig.9. Feature settings in NI-LabVIEW ECG Feature Extractor

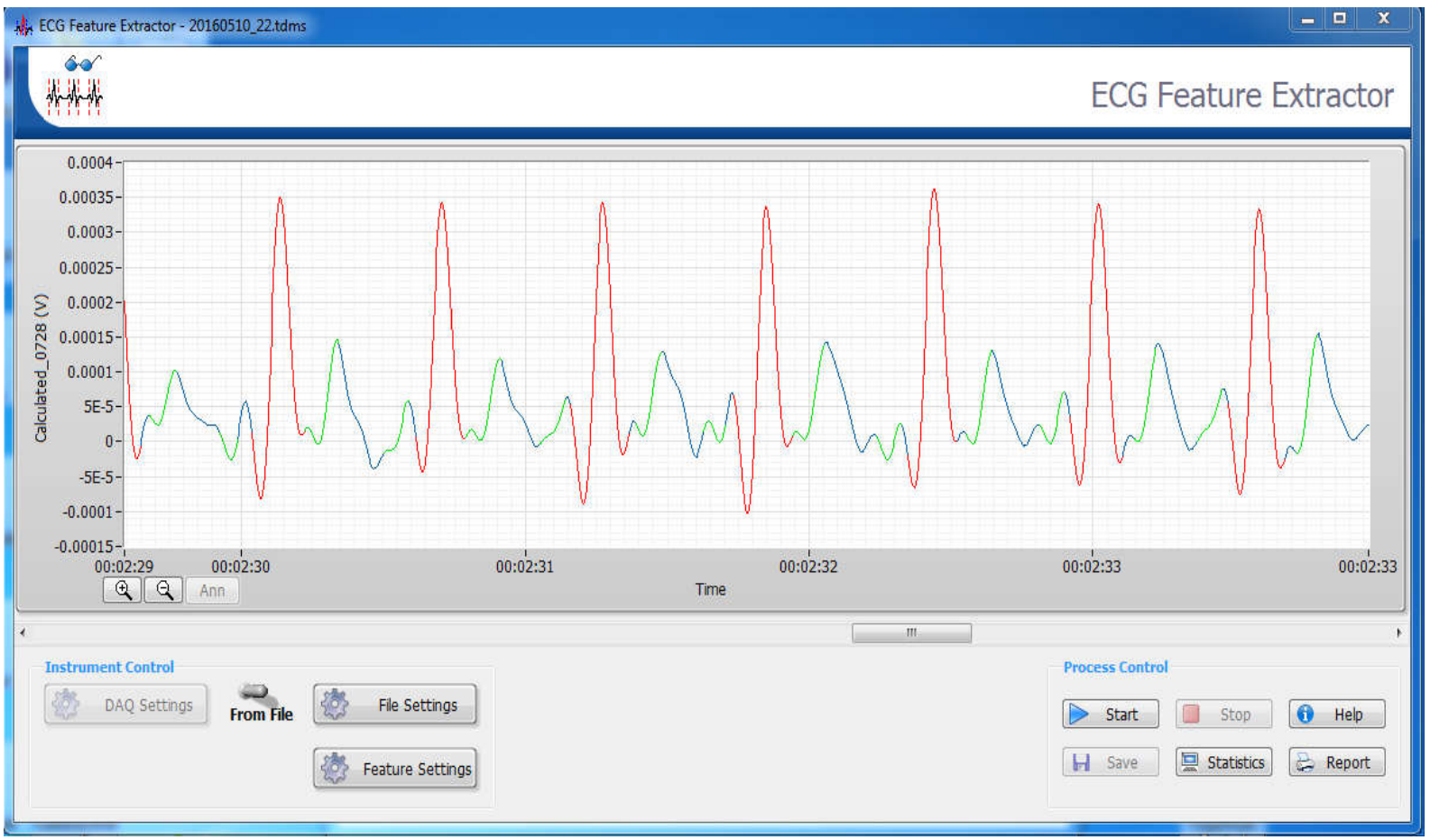

Fig.10. Filtered ECG signals with bandpass frequency $10-25 \mathrm{~Hz}$ 


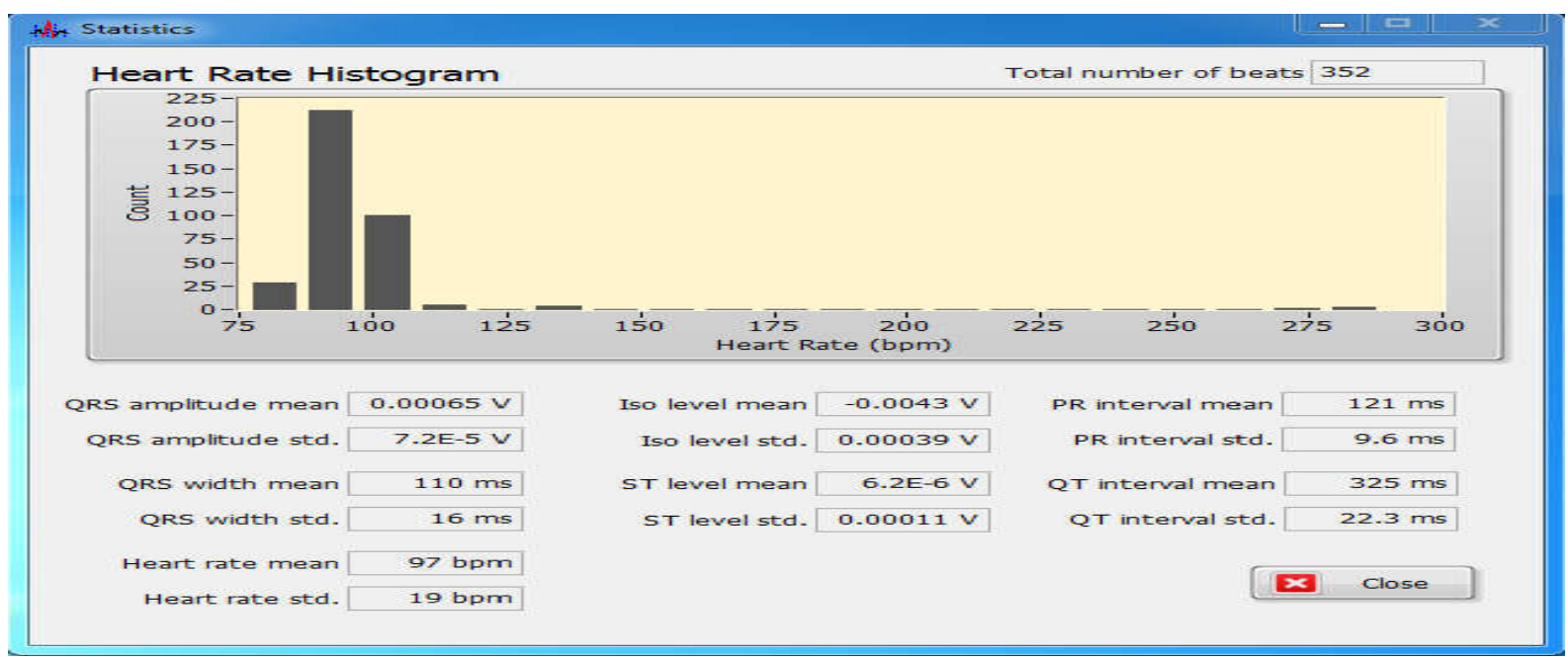

Fig.11. All extracted ECG features are displayed

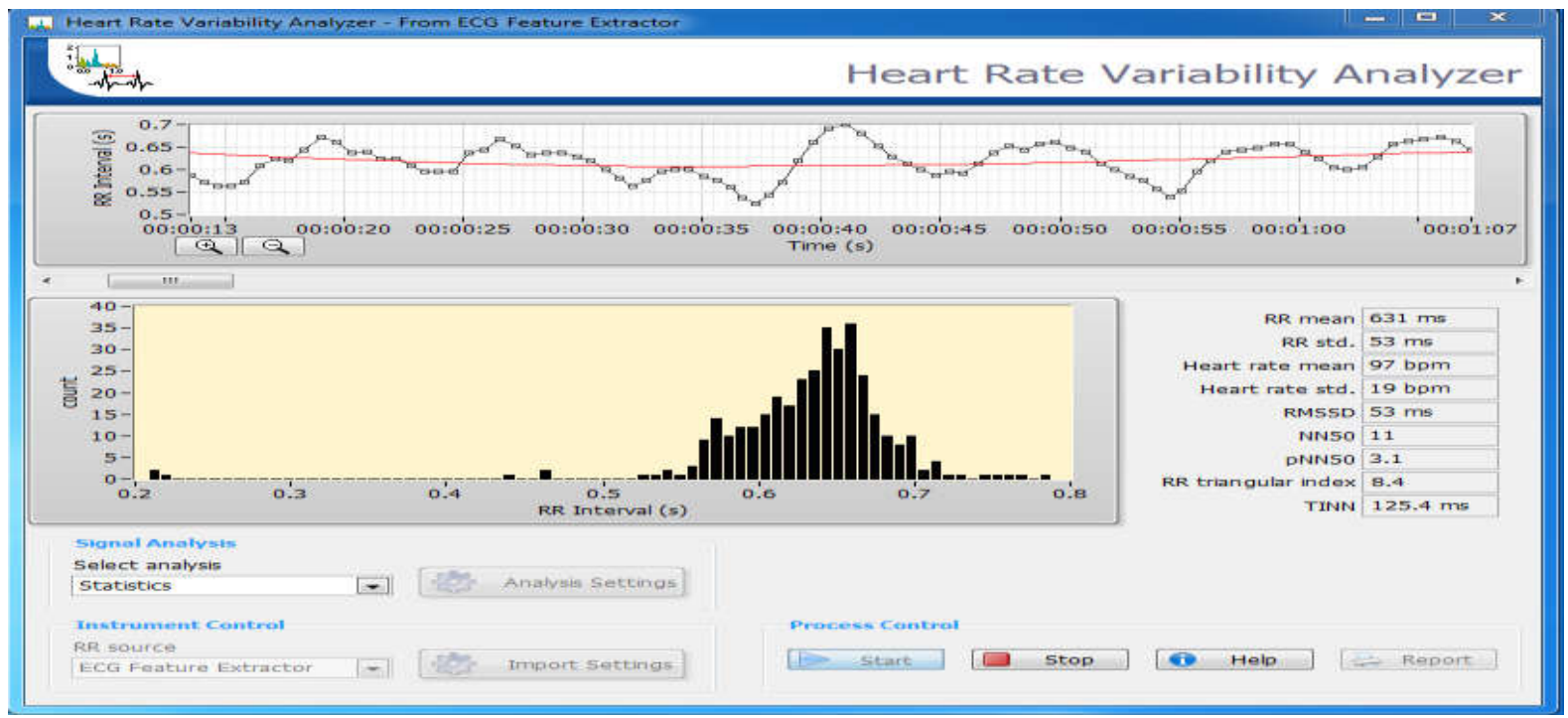

Fig.2. Heart rate variability analyzer application provided by NI-LabVIEW Biomedical Workbench is used to demonstrate the state of the heart and nerve system The bulky and expensive conventional ECG machines used in health facilities are hard to travel along with the militaries and only a piece of ECG paper with ECG signals is available to be obtained. Hence, it is difficult for the militaries to check their cardiovascular conditions when sudden CVD incidents are happen. In order to provide an alternative method for militaries to regularly check their cardiovascular conditions, NI-LabVIEW Biomedical Workbench is presented in this study to obtain and extract ECG signals for health monitoring purposes. Although ECG test can precisely demonstrate the state of heart, but it requires the electrodes attached to human skin are connected to ECG machines using lead wires for gathering the bioelectrical generated by heart. Hence, ECG test is inconvenient to conduct 
when militaries are performing a task or under training sessions due to the limited mobility caused by the wired electrodes. It is more preferable to conduct the ECG test when militaries are at rest. Hereby, a noncontact method of using video imaging technique is proposed in this study to measure heart beat rate. If the militaries in deployment or other activities being detected with irregular or abnormal heart beat rate through video imaging technique, they can perform ECG test immediately with NI-LabVIEW Biomedical Workbench and transmit the ECG signals to military surgeon for examining without travel to health facilities. Besides, militaries can also obtain the information regarding their heart and nerve systems on the spot by using the NI-LabVIEW Biomedical Workbench.

Heart is the main organ in human cardiovascular system to pump blood throughout the human body. When blood circulates human body, tissues can receive oxygen and nutrients. Besides, blood can also bring the metabolic waste away from human. Human blood consists of blood cells (red and white), plasma and platelets. Hemoglobin in red blood cells is the pigment which has light absorption ability. In [36] claimed that the absorption spectrum of hemoglobin is associates with wavelength. Fig. 11 indicates the relations of spectral absorption of hemoglobin from $400 \mathrm{~nm}$ to $700 \mathrm{~nm}$, which is the range of wavelength for visible light [37]. Fig. 13 shows that hemoglobin is greatly absorbed the green color wavelength $(550 \mathrm{~nm})$, if compared to red $(650 \mathrm{~nm})$ and blue $(450 \mathrm{~nm})$ color wavelength. Due to this fact, green color channel of the ordinary camera with RGB sensors supposes can be used to measure the volumetric change of blood flows underneath of the human skin. Based on these volumetric changes of the blood which caused by the heart contraction, cardiovascular parameters such as heart beat rate and blood pressure are possible to be obtained through video imaging technique. In this study, heart beat rate extraction technique through video imaging technique is demonstrated. Video imaging technique which is noncontact, low cost, multiple people assessment and continuous monitoring had made it suitable to be used in detecting militaries heart beat rate when they are performing task or trainings. If irregular or maximum heart beat rate being detected on militaries, they can be stopped from their duty by performing a full ECG diagnosis in order to reduce the chance of CVD happen. 


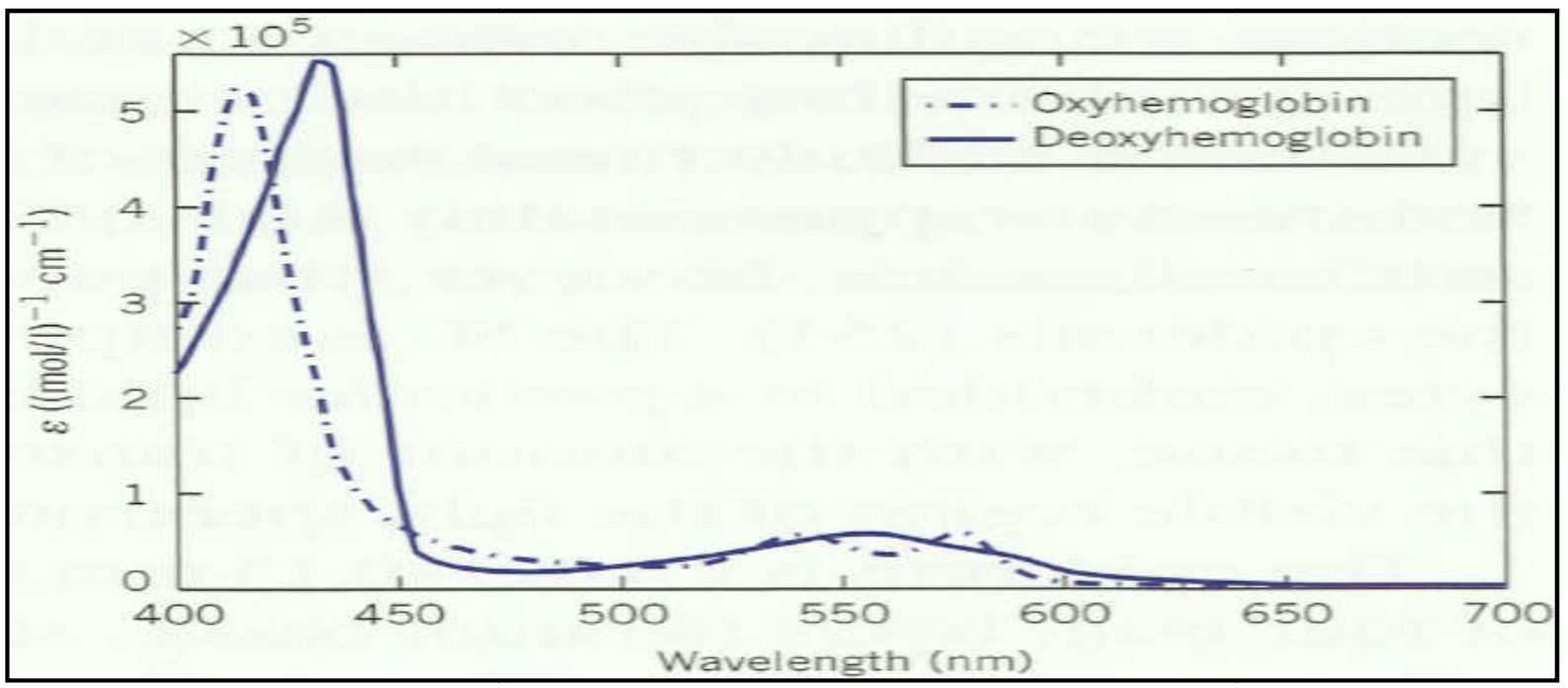

Fig.13. Spectra absorption of hemoglobin from 400-700nm wavelength [37]

In this study, a camera is used to record video of the subject for the heart beat rate analysis. Specimen videos are recorded in RGB color at $30 \mathrm{fps}$ with pixel resolution of $845 \times 480$ pixels. The duration of each recorded video specimens is 60 seconds. Nine sets of video with participants between ages 13-81 years old (5 males and 4 females) are studied to test the reliability in obtaining heart beat rate through video imaging. The recorded video is analyzed by using MATLAB-R2013a platform. The region of interest $(\mathrm{ROI})$ with $50 \times 20$ pixels resolutions of human skin surface are used to extract the heart beat rate.

An image is formed when illumination on the surface of the target objects is reflected to the camera sensors. According to [37], approximately of 5\%-7\% of the light incidents on the skin surface are reflected back to the environment, meanwhile the portion of light that is not reflected by the skin surface are transmitted into the internal skin layers. Subsequently, pigments i.e. melanin and hemoglobin will absorb the incident light transmitted into skin layers and the residues of the absorption are re-emitted back to the skin surface. Since sensors implemented in RGB camera only able to detect the remittance spectra from the skin surface, and hence the image formed are consists of the light that directly reflected by the skin surface and the in-vivo residues of light that re-emitted back to the skin surface. Filtering on these captured RGB signals are able to provide valuable physiological parameters. However, the skin surface is considered inhomogeneous.Any small movement will cause large changes on the remittance spectra captured by the RGB camera, thus ROI is used instead of a single pixel point for heart beat rate extraction. As stated earlier, hemoglobin is strongly absorbed the light 
with a wavelength of $550 \mathrm{~nm}$. Therefore, green color component of the image can be used to determine the blood volume changes in blood vessels. Based on these volumetric changes in blood, subject's heart beat rate can be estimated.

The captured video is in the form of RGB, hence the pixels value in the green color channel of ROI are averaged and normalized to remove the effects of intensity variations within the ROI through Equation (2) where $r, g$ and $b$ are red, green and blue color value respectively and $i$ is the frame index. The normalized values from green color channel show a linear trend that must be removed because it can obstruct the data for analysis. Hence, linear detrend function is used to eliminate the linear trend from the normalized green color values. The detrend function helps to emphasis on analyzing the fluctuations in the data about the trend and it also the preprocessing step usually for Fast Fourier Transform (FFT), which is used to indicate the heart beat rate. Fig. 14 shows the green color pixels' values of ROI and normalized green color signals before and after the detrend function. From the figure, the linear trend is removed and the baseline of the signals will be at origin.

$g_{i}=\frac{g_{i}}{\sqrt{\left(r_{i}\right)^{2}+\left(g_{i}\right)^{2}+\left(b_{i}\right)^{2}}}(2)$

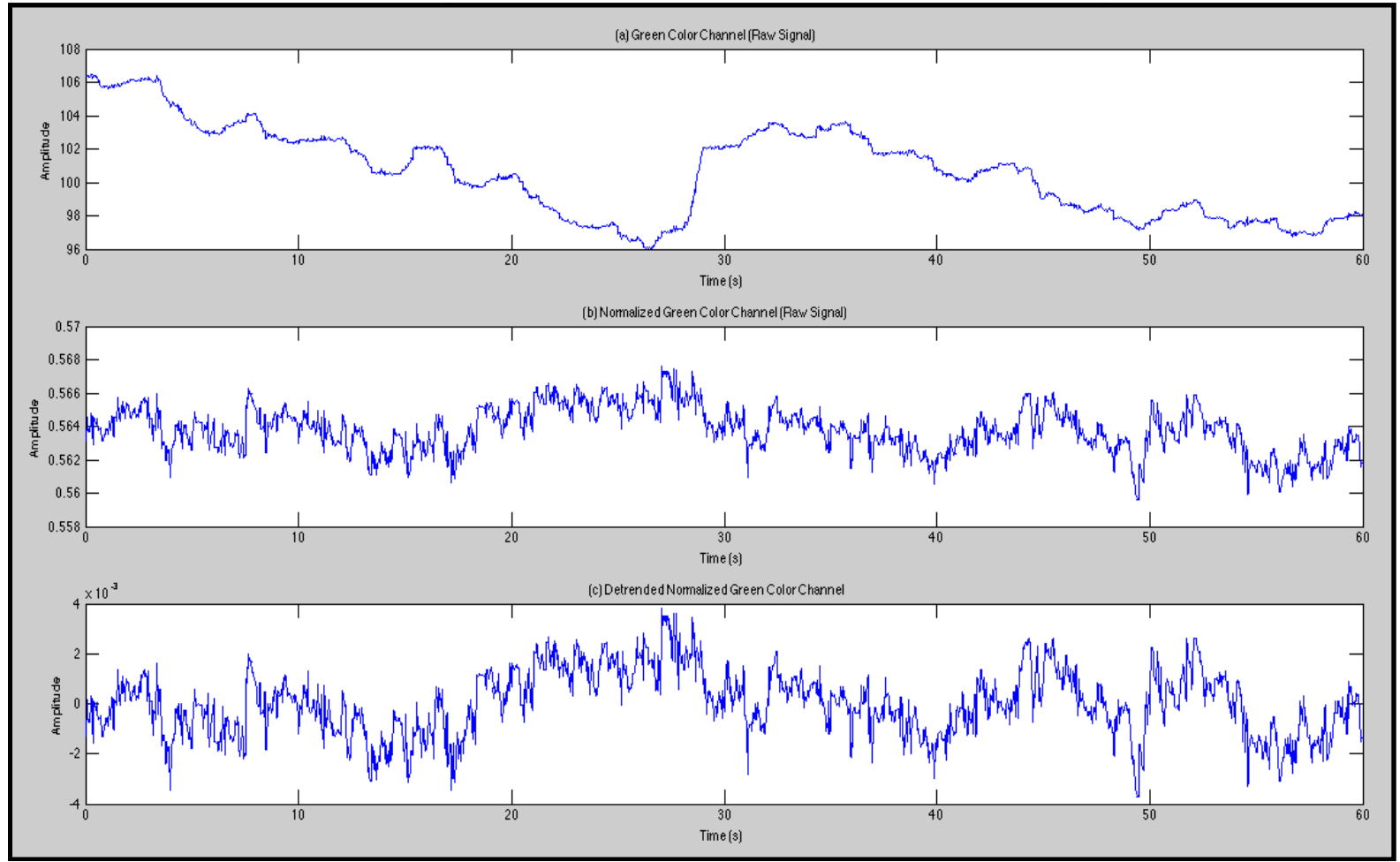

Fig.34. (a) Green color pixel values on ROI, (b) and (c) normalized green color signals before and after detrend respectively 
After the normalized green color signals being detrended, the signals are filtered with fourth-order Butterworth filter with bandpass frequencies $0.7 \mathrm{~Hz}-4 \mathrm{~Hz}$ to further attenuate the signal noises. The frequency was set at $0.7 \mathrm{~Hz}-4 \mathrm{~Hz}$ due to the fact that heart beat rate for a normal person is within the range of $42 \mathrm{bpm}-240 \mathrm{bpm}$. After the signals being filtered, the signals are transformed into frequency domain using FFT to extract the heart beat rate.

The recorded video with frames rate of $30 \mathrm{fps}$ are sequence of images in time, changes of the green color intensities between consecutive images over time represent a series of signals that contain the information of the blood volume changes under the skin surface. In order to extract the heart, beat rate from video imaging, frequency domain is needed for the spectral analysis. Discrete Fourier Transform (DFT) is the most usually used algorithm to transform the signals in time domain to the signals in frequency domain. In this study, Fast Fourier Transform (FFT) is used because of its faster computational time when computing the DFT. In order to provide a continuous estimation of the heart beat rate, a window length of 6 seconds with $91.2 \%$ overlaps between adjacent windows is slides over the entire recorded video signals. The interval between the consecutive sliding windows is 0.5 seconds. In fact, the larger the window length able provides a better frequency resolution which is important for the computational accuracy. However, increasing the window length will decrease the time accuracy. For instance, if the entire signal length is selected as the window length, it is impossible to precisely identify the frequency when the signals are started and how long the signals are sustained. Thus, sliding window is normally applied to diminish the temporal autocorrelation [38]. Temporal autocorrelation occurs when the measurements of a time series are influenced by its preceding values. The signals in the sliding window are converted to Power Spectral Density (PSD) for extracting the frequency with the highest power spectral. Equation (3) is used to determine the subject's heart beat rate and Equation (4) is used to calculate the mean value of the heart beat rate for the entire recorded signals where $S H B$ is the heart beat rate of 6 seconds sliding window, $f$ is the frequency with highest peak, $k$ is the index of sliding windows, $N$ is the total number of sliding windows and $B P M$ is the mean value of heart beat rate in 1 minute. Fig. 15 shows the procedures to extract the heart beat rate through video imaging technique. 
$S H B(k)=f(k) * 60$
$B P M=\frac{1}{N} \sum_{k=1}^{N} S H B(k)(4)$

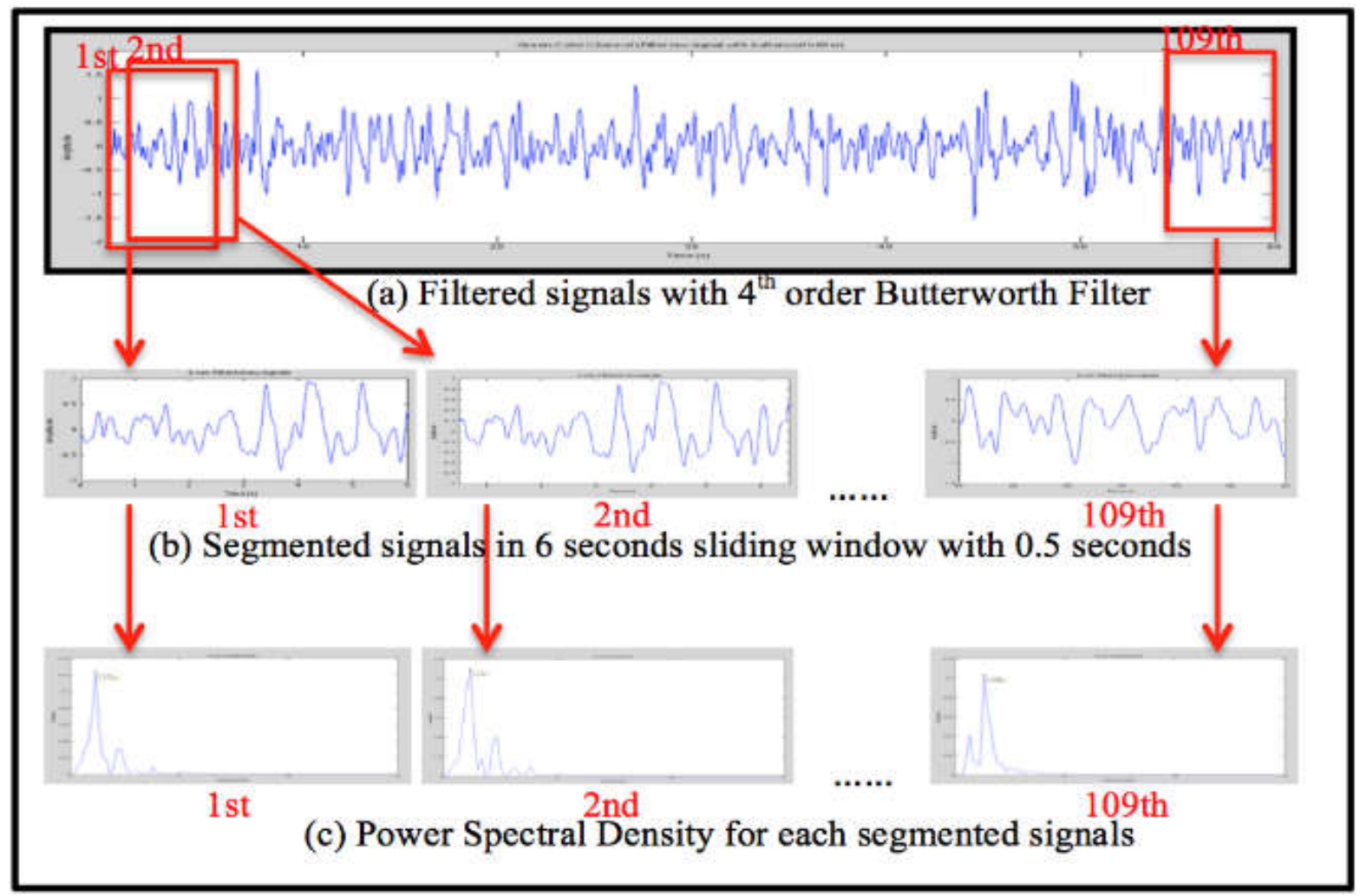

Fig.45. The procedures to extract heart beat rate through video imaging technique

\section{CONCLUSION}

In this study, NI-LabVIEW Biomedical Workbench with DAQ device is presented to extract various ECG features. The extracted features through NI-LabVIEW are compared to the features extracted from the ECG machine used in health facilities, which model is Philips PageWriter TC30. The results show that the ECG features extracted through NI-LabVIEW Biomedical Workbench are comparable and reached a good agreement with the standard ECG machines. Hence, it is possible becomes an alternative method to extract ECG feature for detection of CVD. Besides, NI-LabVIEW Biomedical Workbench also provides various applications on physiological parameters analysis for the users to analyze their health conditions immediately without travel to health facilities especially for the militaries that always deployed in the battlefield, mountain, ocean or forest which normally distant from health facilities. The computer-based NI-LabVIEW with self-interpretation functions is very 
beneficial than the conventional ECG machines that require cardiologist or professional to interpret the ECG features printed on the ECG paper. Moreover, NI-LabVIEW Biomedical Workbench can store the ECG signals permanently for replaying and checking their previous recorded cardiovascular conditions. Besides, the soft copy can also transmit to the surgeon in advance for early preparation of the appropriate facilities when the patient is on the way to the health facilities. As a conclusion, NI-LabVIEW Biomedical Workbench with various ECG features extraction is suitable to be applied for military to monitor their health status regularly. Although ECG test can precisely in determining heart activities, but it requires attached the wired electrodes on the human body which can cause inconvenient. Thus, video imaging technique is proposed to extract the heart beat rate remotely. If the on-duty military with irregular, unusual or maximum heart beat rate being detected, he or she can be requested to perform a full scan of the ECG test through NI-LabVIEW Biomedical Workbench immediately. From the results, it shows that heart beat rate extractions through video imaging are comparable and correspond with the results obtained from NI-LabVIEW Biomedical Workbench. Although the largest difference in the mean value of heart beat rate is reached 6 bpm with $9.4 \%$ of readout error for the subject at age 81 years old, but it still fulfill the requirements that stated in the American National Standard ANSI/AAMI EC13:2002 which developed by [39]. Hence, video imaging can be used to extract the heart beat rate for early detection of CVD due to its advantages of noncontact, low cost, multiple people assessments and continuous monitoring.

\section{ACKNOWLEDGEMENTS}

The authors would like to acknowledge the financial assistance from $\mathrm{MyPhD}$ under MyBrain15 and Fundamental Research Grant Scheme (FRGS) with grant no. FRG0350-TK-2/2013 by Ministry of Higher Education (MoHE). Authors would also like to thank Centre for Postgraduate Studies, Universiti Malaysia Sabah for the funding provided and Polyclinic Universiti Malaysia Sabah to provide the facilities in conducting ECG test. 


\section{REFERENCES}

[1] Pollock PH. Eye movement desensitization and reprocessing (EMDR) for post-traumatic stress disorder (PTSD) following homicide. Journal of Forensic Psychiatry, 2000, 11(1):176-184

[2] Reuters. One in 12 U.S. soldiers who died in combat had plaque buildup in their arteries that is early sign of heart disease. London: Dailymail Online, 2012

[3] Perk J, De Backer G, Gohlke H, Graham I, Reiner Ž, Verschuren WM, Albus C, Benlian P, Boysen G,Cifkova R, Deaton C. European guidelines on cardiovascular disease prevention in clinical practice (version 2012). International Journal of Behavioral Medicine, 2012, 19(4):403-488

[4] Naghavi M, Libby P, Falk E, Casscells S W, Litovsky S, Rumberger J, Badimon J J, Stefanadis C, Moreno P, Pasterkamp G, Fayad Z, Stone P H, Waxman S, Raggi P, Madjid M, Zarrabi A, Burke A, Yuan C, Fitzgerald P J, Siscovick D S, C. L. de Korte, Aikawa M, Airaksinen K E J, Assmann G, Becker C R, Chesebro J H, Farb A, Galis Z S, Jackson C, Jang I K, Koenig W, Lodder R A, Mearch K, Demirovic J, Navab M, Priori S G, Rekhter M D, Bahr R, Grundy S M, Mehran R, Colombo A, Boerwinkle E, Ballantyne C, Insull W, Schqartz R S, Vogel R, Serruys P W, Hansson G K, Faxon D P, Kaul S, Drexler H, Greenland P, Muller J E, Virmani R, Ridker P M, Zipes D P, Shah P K,Willerson J T. From vulnerable plaque to vulnerable patient-A call for new definitions and risk assessment strategies: Part II. Circulation, 2003, 108:1772-1778

[5] Poirier P, Dufour R, Carpentier A, Larose E. Screening for the presence of coronary artery disease. Canadian Journal of Diabetes, 2013, 37:105-109

[6] Roger V L, Go A S, Lloyd-Jones D M, Benjamin E J, Berry J D, Border W B, Bravata D M, Dai S, EFord E S, Fox C S, Fullerton H J, Gillespie C, Hailpern S M, Heit J A, Howard V J, Kissela B M, Kittner S J, Lackland D T, Lichtman J H, Lisabeth L D, Makuc D M, Marcus G M, Marelli A, Matchar D M, Moy C S, Mozaffarian D, Mussolino M E, Nichol G, Paynter N P, Soliman E Z, Sorlie P D, Sotoodehnia N, Turan T N, Virani S S, Wong N D, Woo D, Turner M B. Heart disease and stroke statistics-2012 update: A report from American Heart Association. Circulation, 2012, 125(1):45-53 
[7] Lin $\mathrm{W} \mathrm{H}$, Zhang $\mathrm{H}$, Zhang $\mathrm{Y}$ T. Investigation on cardiovascular risk prediction using physiological parameters. Computational and Mathematical Methods in Medicine, 2013,2013: $1-21$

[8] Wilson D R,Folkes F. Cardiovascular disease in military populations: Introduction and overview. Journal of the Royal Army Medical Corps, 2015, 161(3):167-168

[9] Moses D,Deisy C. A survey of data mining algorithms used in cardiovascular disease diagnosis from multi-lead ECG data. Kuwait Journal of Science, 2015, 42(2):206-235

[10] Hadjem M, Salem O,Nait-Abdesselam F. An ECG monitoring system for prediction of cardiac anomalies using WBAN. In IEEE 16th International Conference on e-Health Networking, Applications and Services, 2014, pp. 441-446

[11] Peng R C, Zhou X L, Lin W H, Zhang Y T. Extraction of heart rate variability from smartphone photoplethysmograms. Computational and Mathematical Methods in Medicine, $2015,2015: 1-12$

[12] Subramanian K, Thaker J, Savla P, Rane M. Smart solar ECG. In International Conference on Electrical, Electronics, Signals, Communication and Optimization, 2015, pp. $1-5$

[13] Lim H W, MohdSani M S A, Hashim A,Hau Y W. Throb: System-on-chip based arrhythmia screener with self interpretation. International Journal of Electrical and Electronic Systems Research, 2015, 8:30-36

[14] Oweis R J, Al-Tabbaa B O. QRS detection and heart rate variability analysis: A survey. Biomedical Science and Engineering, 2014, 2(1):13-34

[15] Babu M, Raju R R, Sylvester S, Mathew T M,Abubeker K M. Real time patient monitoring system using LabVIEW. International Journal of AdvancedResearch in Computer and Communication Engineering, 2016, 5(3):505-507

[16] Quintana D S, Guastella A J, Outhred T, Hickie I B, Kemp A H. Heart rate variability is associated with emotion recognition: Direct evidence for a relationship between the autonomic nervous system and social cognition. International Journal of Psychophysiology, 2012, 86(2):168-172.

[17] Beasley R. M. Understanding EKGs: A practical approach. New Jersey: Prentice Hall, 
2003

[18] Brisendine K. J. Multiskilling: Electrocardiography for the health care provider. New York: Delmar Publishers, 1998

[19] Al-Ziarjawey H A J,Cankaya I. Heart rate monitoring and PQRST detection based on graphical user interface with MATLAB. International Journal of Information and Electronics Engineering, 2015, 5(4):311-316

[20] Eggett C, Eldridge J,Richley D. Clinical guidelines by concensus recording a standard 12-lead electrocardiogram: An approved methodology by the Society for Cardiological Science and Technology (SCST). Technical report, Staffordshire: SCST, 2014

[21] Sydney Children's Hospitals Network (SCHN). Continuous electrocardiography (ECG) monitoring-SCH Practice Guideline. Westmead: SCHN, 2013

[22] Sydney Children's Hospitals Network (SCHN). Electrocardiographic (ECG) monitoring-CHW procedure. Westmead: SCHN, 2014

[23] Barrett K. E., Barman S. M., Boitano S., Brooks H. L.Ganong'sreview of medical physiology. New York: McGraw Hill Education, 2012

[24] Vaitkunas A D M, Daunoras J. Measurement of human physiological parameters in the systems of active clothing and wearable technologies. Electronics and Electrical Engineering, 2006, 7(71):77-82

[25] Halvorsen H P. Tutorial: Data acquisition in LabVIEW. Kongsberg: University College of Southeast Norway, 2016

[26] National Instruments (NI). NI-USB 6281 datasheet.Texas: NI, 2014

[27] National Instruments (NI). DAQ M series: M series user manual-NI 622x, NI 625x, and NI 628x devices.Texas: NI, 2007

[28] Deshmukh A,Gandole Y. ECG feature extractor using NI Lab-VIEW biomedical workbench. International Journal of Recent Scientific Research, 2015, 6(8):5603-5607

[29] Balakrishnan G, Durand F,Guttag J. Detecting pulse from head motions in video. In IEEE Conference on Computer Vision and Pattern Recognition, 2013, pp. 3430-3437 [30] National Instruments (NI). LabVIEW for ECG signal processing. Texas: NI, 2012 [31] Islam M K, Haque A N H M, Tangim G, Ahammad T,Khondokar M R H. Study and 
analysis of ECG signal using MATLAB and LABVIEW as effective tools. International Journal of Computer and Electrical Engineering, 2012, 4(3):404-408

[32] Shetty P, Bhat S. Analysis of various filter configurations on noise reduction in ECG waveform. International Journal of Computing, Communications and Instrumentation Engineering, 2014, 1(1):88-91

[33] National Instruments (NI). How to use LabVIEW biomedical toolkit-ECG feature extractor. Texas: NI, 2012

[34] Srinagesh M, Sarala P,Aparna K D. ECG wireless telemetry. International Journal of Engineering and Innovative Technology, 2013, 2(8):75-78

[35] Elmansouri K, Latif R, Nassiri B,Elouaham S. New Electrocardiogram signal analysis in a research laboratory using LabVIEW. International Journal of Interdisciplinary Research and Innovations, 2013, 1(1):1-7

[36] Liu Z,Zerubia J. Melanin and hemoglobin identification for skin disease analysis. In Asian Conference on Pattern Recognition, 2013, pp. 1-5

[37] Baranoski G. V. G.,Krishnaswamy A. Light andskin interactions: Simulations for computer graphics applications. Massachusetts: Morgan Kaufmann Publishers, 2010

[38] Chang C, Liu Z M, Chen M C, Liu X,Duyn J H. EEG correlates of time-varying BOLD functional connectivity. Neuroimage, 2013, 72:227-236

[39] Association for the Advancement of Medical Instrumentation (AAMI). American National Standard ANSI/AAMI EC13:2002: Cardiac monitors, heart rate meters, and alarms. Virginia: AAMI, 2002

[40] Poh M Z, McDuff D J, Picard R W. Non-contact, automated cardiac pulse measurements using video imaging and blind source separation. Optics Express, 2010, 18(10):10762-10774 [41] NorM N M, Jailani R, TahirN M, Ihsan M Y, Zairi I R, Rahmat H.EMG signals analysis of $\mathrm{BF}$ and $\mathrm{RF}$ muscles in autism spectrum disorder (ASD) during walking. International Journal on Advanced Science, Engineering and Information Technology, 2016, 6(5):793-798

\section{How to cite this article:}

Khong W L, Rao N S V K, Mariappan M. National instruments labview and video imaging technique for health status monitoring. J. Fundam. Appl. Sci., 2017, 9(3S), 858-886 